\title{
Observations of Variable Stars by the 76-cm SuperLight Telescope of NCU
}

\author{
J. Z. Li ${ }^{1,2}$,C.H. Wu ${ }^{1}$,Z.W. Zhang ${ }^{1}$,C.P. Chang ${ }^{1}$, C.Y. Lin ${ }^{1}$, H.H.

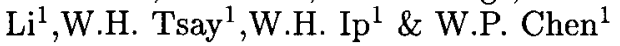 \\ 1. Institute of Astronomy, National Central University, Chung-Li 32054 \\ 2. Beijing Astronomical Observatory, National Astronomical \\ Observatory, Chinese Academy of Sciences, Beijing 100012, China \\ (Email: ljz@astro.ncu.edu.tw)
}

\begin{abstract}
The self-constructed Super-Light telescope of the National Central University is now ready for open use. Systematic studies of RR Lyraes stars and other variables are outlined and some preliminary results are introduced.
\end{abstract}

\section{Introduction}

During the last several months, the functionality of the newly constructed $76 \mathrm{~cm}$ Super-Light Telescope of the Institute of Astronomy has been considerably improved. This small telescope has basically passed its testing phase and is now ready for scientific data acquisition. Light-variation of some CVS and RR Lyrae variables, obtained during the test mode observations of the telescope, is presented in this paper.

\section{SLT Program of Variable Stars}

- Study of some newly identified CVs by differential photometry

- Photometric study of a sample of unusual RR Lyrae stars from the Hipparcos Catalogue.

- Identification and study of a sample of RR Lyrae candidates from SDSS

- Monitoring of X-ray binaries as a part of our routine observations

\section{Preliminary Results from the Test Mode Observations}




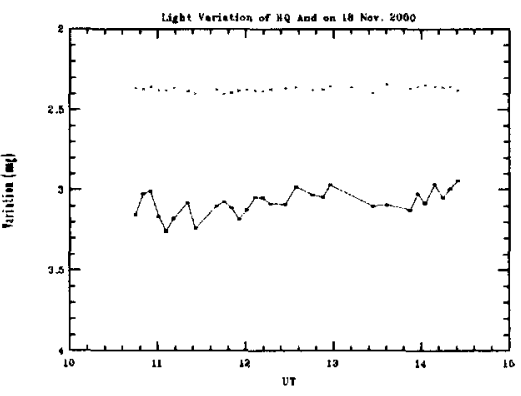

Figure 1. Light variations of a cataclysmic variable $\mathrm{HQ}$ And 15.3mag. (The time resolution of this run of observations of the CV is not high enough to resolve the rapid variations of the $C V$ ).
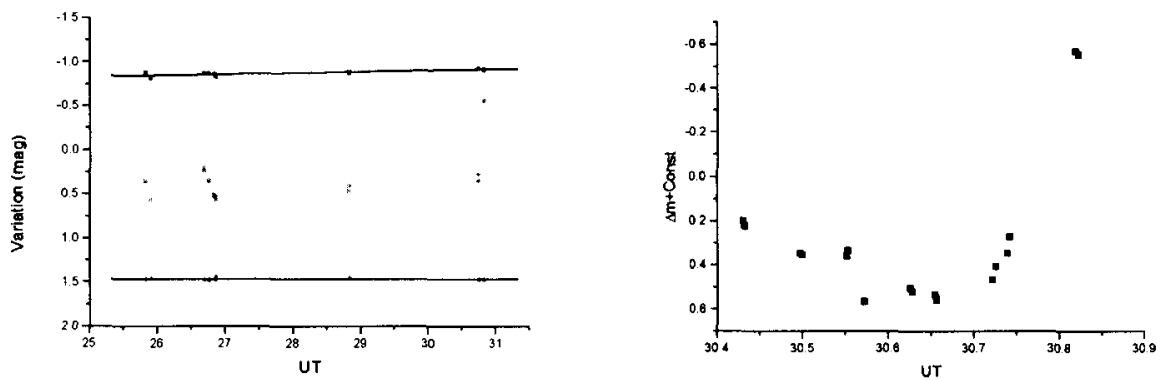

Figure 2. Composite light curve of an unusual RR Lyrae star, AA $\mathrm{Cmi}$, from the fit of several days' discontinuous observations.
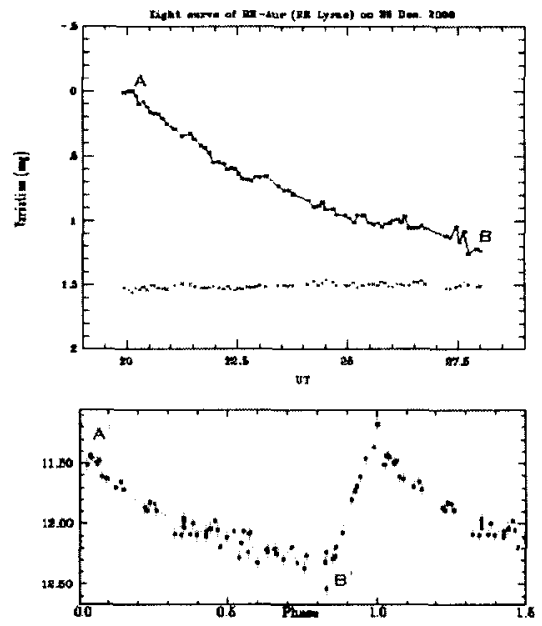

Figure 3. The light curve of another unusual RR Lyrae star, BH Aur, based on SLT observations (upper panel), as compared to data from the Hipparcos catalogue (lower panel). 